\title{
1 A survey of Chinese pig farms and human healthcare isolates reveals separate human
}

\section{2 and animal MRSA populations}

3 Geng ZOU ${ }^{1}$, Marta MATUSZEWSKA ${ }^{2}$, Ming JIA ${ }^{1}$, Jianwei ZHOU ${ }^{1}$, Xiaoliang BA $^{2}$, Juan DUAN ${ }^{1}$, Caishi

$4 \mathrm{ZHANG}^{3}$, Jian $\mathrm{ZHAO}^{1}$, Meng TAO ${ }^{1}$, Jingyan $\mathrm{FAN}^{1}$, Xiangming $\mathrm{ZHANG}^{4}$, Wenping $\mathrm{JIN}^{4}$, Tianpen $\mathrm{CUI}^{5}$,

5 Xianyu ZENG ${ }^{5}$, Min JIA $^{5}$, Xiaojuan QIAN $^{6}$, Chao HUANG $^{1}$, Wenxiao ZHUO ${ }^{1}$, Zhiming YAO ${ }^{1}$, Lijun

6 ZHANG ${ }^{1}$, Shaowen $\mathrm{LI}^{1}$, Lu LI ${ }^{1,7,8}$, Qi HUANG ${ }^{1,7,8}$, Bin WU ${ }^{1,7,8}$, Huanchun $\mathrm{CHEN}^{1,7,8}$, Alexander W. TUCKER ${ }^{2}$,

7 Andrew J. GRANT², Mark A. HOLMES ${ }^{2 *} \&$ Rui ZHOU ${ }^{1,7,8^{*}}$.

$8 \quad{ }^{1}$ State Key Laboratory of Agricultural Microbiology, Huazhong Agricultural University College of Veterinary

9 Medicine, Wuhan 430070, China;

$10{ }^{2}$ Department of Veterinary Medicine, University of Cambridge, Cambridge, UK;

$11{ }^{3}$ Jianli People's Hospital, Jianli 433300, China;

$12 \quad{ }^{4}$ Wuhan Third Hospital, Wuhan 430060, China;

$13{ }^{5}$ Wuhan First Hospital, Wuhan 430014, China;

$14{ }^{6}$ Huangmei People's Hospital, Huangmei 435500, China

$15{ }^{7}$ Cooperative Innovation Center of Sustainable Pig Production, Wuhan 430070, China

$16{ }^{8}$ International Research Center for Animal Diseases (MOST), Wuhan 430070, China

17

18 *Corresponding authors: rzhou@ @ail.hzau.edu.cn (Rui Zhou); mah1@cam.ac.uk (Mark A. Holmes) 


\section{Abstract}

20 There has been increasing concern that the overuse of antibiotics in livestock farming is contributing to the 21 burden of antimicrobial resistance in people. Farmed animals in Europe and North America, particularly pigs, provide a reservoir for livestock-associated methicillin-resistant Staphylococcus aureus (LA-MRSA, ST398) found in people. This study was designed to investigate the contribution of MRSA from Chinese pig farms to human infection and carriage.A collection of 603 S. aureus were isolated from 55 pig farms and 4 hospitals $(\mathrm{MRSA}=285,198 ; \mathrm{MSSA}=50,70)$ in central China, a high pig farming density area, during 2017-2018. CC9 MRSA accounting for 93\% of all farm MRSA isolates, while no was found in hospitals. ST398 isolates were found on three farms $(n=23)$ and three hospitals $(n=12)$. None of the ST398 from this study belong to the livestock clade of the LA-MRSA commonly found in Europe and North America. The hospital ST398 MRSA isolates formed a clade that was clearly separate from the farm ST398 MRSA and MSSA isolates, and all possessed human immune evasion cluster genes which were absent from all the pig farm ST398 isolates. Despite the presence of high levels of MRSA found on Chinese pig farms we found no evidence of them spilling over to the human population. Nevertheless, the ST398 MRSA obtained from human samples appear to be part of a widely distributed lineage in China. And the new animal adapted ST398 lineage that emerged in China should also be alarmed.

\section{Importance}

37 We disclosed the fact that although the high MRSA positive rate in Chinese hospitals and pig farms should be alarmed, they might be two separate issues. The new CC398 clades we identified highlight that the host adaption of the MRSA lineage is kept changing. These results suggest that continued surveillance of MRSA in livestock is necessary. We found that the pig farm MRSA isolates had unique antimicrobial resistance genes while most of the hospital MRSA isolates had human immune evasion cluster genes. These features could be used to distinguish the pig farm associated S. aureus in clinical laboratories. The policies of reducing antimicrobials use in livestock were implemented in China since 2020. Our study described the situation of MRSA populations in to evaluate the effects of the policies. 


\section{Introduction}

47 Staphylococcus aureus (S. aureus) is an opportunist human pathogen that can also colonize and infect many 48 species of animals and adapt to diverse environmental conditions ${ }^{1}$. Methicillin-resistant S. aureus (MRSA) are

49 S. aureus lineages that have acquired the mecA or mecC genes through horizontal gene transfer which makes

50 them highly resistant to nearly all $\beta$-lactam antibiotics ${ }^{2}$.

51 The zoonotic potential of MRSA in livestock and wild animals has been widely reported ${ }^{3}$. An early report of 52 livestock associated MRSA (LA-MRSA) described an infection in a baby living on a farm where the pigs were 53 infected with MRSA Sequence Type (ST)398, in the Netherlands in $2005^{4}$. This LA-MRSA lineage is now widely distributed in pigs and other farm animal populations in Europe and North America ${ }^{5,6}$. A recent national report of AMR in Denmark gave the prevalence of LA-MRSA in finishing pigs in randomly selected herds as $88 \%$ with ST398 accounting for $16 \%$ of all human MRSA infections which raises considerable concerns about antibiotic stewardship in agriculture and the threat to public health ${ }^{7}$.

Phylogenetic studies have shown that LA-MRSA ST398 originated from a human MSSA lineage and jumped to livestock, acquiring tetracycline and methicillin resistance ${ }^{8,9}$. These two clades can be distinguished on the basis of canonical single-nucleotide polymorphisms (SNPs) ${ }^{10}$.

61 The LA-MRSA clonal lineage most frequently reported in Asian livestock is ST9 with closely related isolates from people and livestock reported in the literature ${ }^{11,12}$. This ST is also common in European and North American pig farms almost exclusively as a MSSA. The origins of this lineage are unclear although it is common in historic collections of livestock isolates suggesting an animal origin ${ }^{13,14}$. In addition to ST398 and ST9 MRSA, among other lineages (CC1, CC5, CC8, CC59, CC97, CC130 and CC425) have been found in livestock with evidence that they are also involved in zoonotic or anthroponotic transmission (host switching) ${ }^{15,16}$.

Previous studies looking at LA-MRSA in China have shown that ST9 is the dominant lineage in pigs with

69 ST398 MRSA being less commonly detected ${ }^{17,18,19}$. Studies of human isolates have identified ST398 MRSA as a cause of significant disease in patients from Chinese hospitals ${ }^{20}$. Genomic studies of human isolates have indicated that some of these isolates are from the human ST398 lineage having acquired a mecA gene in a distinctive SCCmec V variant ${ }^{21}$.

73 In order to assess the contribution of pig farms to the human burden of MRSA, a study was designed to enable a genomic study of MRSA isolates from human and animal hosts. Isolates of S. aureus were collected contemporaneously from pig farms and hospitals in the same region in China and subjected to DNA sequencing. 
A phylogenetic analysis was performed in order to establish if the human isolates were likely to be of livestock origin.

\section{Results}

\section{The MRSA populations of pig farms and hospitals consisted of different clonal lineages}

In the samples collected from 55 pig farms (pig nasal swabs $=2416$, farm worker nasal swabs $=361$, pig farm dust samples $=291$ ), 335 S. aureus isolates were identified and sequenced (based on phenotypic antibiotic susceptibility 285 of them were MRSA). MRSA were isolated from 34/55 pig farms (62\%). CC9 MRSA were present in all positive farms where more than one MRSA isolate was obtained and accounted for 97\% (277/285) of all farm MRSA isolates. ST398 isolates were found on 3 farms: one ST398 MRSA was isolated from a farm worker on one farm, two ST398 MRSA isolates were obtained from a pig from another farm, and a number of ST398 MSSA were isolated from pigs, environmental samples and a farm worker on the third farm. The remaining 5 farm MRSA isolates comprised one ST1 (from a pig sample) and 4 ST59 (1 from a pig and 3 from farm worker swabs) (Figure S1, Table S1). 50 MSSA pig farm isolates included 16 ST9, 5 ST1, 4 ST5, 20 ST398, and 5 ST128. (Table 1).

A total of 268 S. aureus isolates were collected from four hospitals $($ MRSA $=198$, MSSA $=70)$ and 39 different STs were identified. The three most common STs in the hospital MRSA populations were ST59 (66/198), ST239 (42/198) and ST45 (17/198). Five ST398 MRSA isolates were identified from three hospitals, including two city hospitals and a county hospital, seven ST398 MSSA isolates were also identified in the county hospital (Table 1). Notably there were no ST9 isolates (MRSA or MSSA) found in the Chinese hospitals.

\section{Phylogenetic analysis identifies separate human and livestock ST398 clades in China.}

Phylogenetic studies can provide evidence about the population structure and rates of transmission between different hosts. Where genomes from different hosts are mixed in each clade, this indicates jumps between host species. Where strains from different hosts form distinct clades, this suggests that distinct populations are being maintained in different hosts, and that host jumps are rare $^{16}$. A phylogenetic analysis was performed to examine the population structure of ST398. A total of 115 Chinese CC398 isolates, including 36 isolates from this study,

10337 isolates collected from NCBI genome database and 42 isolates from hospital patients collected in a previous

104 study ${ }^{21}$ were used to create an alignment against the reference genome 0213-M-4A (a finished pig ST398 105 MRSA genome generated in this study) from which a maximum likelihood tree was constructed (Figure 1). 
106 With the exception of 4 MSSA from pigs in eastern China (Clade C-PL), all the Chinese CC398 MRSA and

107 MSSA isolates (from both livestock and humans) were shown to fall within the diversity of the previously

108 identified 'human' lineage as defined by the published canonical SNPs (Figure 1). The Clade C-PL isolates are

109 contained within the previously identified 'livestock' lineage and carry a tet $\mathrm{M}$ gene which is associated with the

110 'livestock' lineage ${ }^{8}$. All the human hospital MRSA ST398 isolates from this study formed a discrete cluster with

111 other MRSA in the collection from a broad geographical range in China (Clade C-HM). No pig farm (or other

112 animal) isolates contributed to this clade and they all possessed a full set of the human immune evasion cluster

113 genes. The pig farm ST398 MRSA isolates fell within the diversity of another separate clade (Clade C-L) which

114 was comprised of the majority of animal MSSA isolates together with some human MSSA isolates and 5 MRSA

115 (1 farm worker from this study 4 from pigs). All isolates in Clade C-L lacked a full set of the human immune 116 evasion cluster genes and contained a characteristic set of antimicrobial resistance genes (see below). The pig 117 farm MSSA CC398 isolates identified in this study (which all came from a single farm) clustered within Clade

118 C-L. The 8 hospital MSSA CC398 isolates from this study belonged to 2 separate clades (Clade C-H2 and C-

119 H3). Three isolates (JL116, JL108 and JL84) were in the Clade C-H2 separated from most of the other human 120 MSSA. Two (JL42 and JL73) of the other 5 isolates fell in the Clade C-H3-1 containing human hospital MRSA

121 (Clade C-HM) from this study suggesting that SCCmec had been acquired by this human-adapted lineage. Two

122 (JL113 and JL63) of the remaining 3 isolates belonged to the Clade C-H3-2 consisting entirely of human MSSA

123 which had a relatively close relationship to the 'livestock' lineage (Clade C-PL).

124 A time-measured phylogenetic tree using an international collection of ST398 isolates was created using BEAST1.8.2 ${ }^{22}$. After removing all isolates without date, host, and location information in the selection process

126 (see methods), a total of 207 ST398 isolates (11 from this study, and 196 from the NCBI genome collection) were used for the analysis. The collection included isolates collected from around the world over the last 15

128 years. A time annotated tree is shown in Figure 2. The tree shows an ancestral clade at the base of the tree consisting of mainly human MSSA ST398 isolates from Europe and North America, which also includes a

130 Chinese clade (Clade C-H1). The well documented prevalent LA-MRSA clade (the 'livestock' clade; possessing 131 the appropriate canonical SNPs) appears to be the most recently emerged clade. It includes just 2 Chinese 132 isolates (from the Clade C-LP in Figure1) from pigs and is defined by a most recent common ancestor (MRCA) 133 dating back to 1964 (95\% highest posterior density interval [HPD] = [1956, 1972]). With a MRCA estimate of $1341940(95 \% \mathrm{HPD}=[1926,1954])$ the vast majority of Chinese isolates and the 'livestock' clade emerged from the ancestral ST398 clade. Three Chinese clades (Clade CH2, C-L and C-H3) all appear to separate at the same 
136 time with the MRCA dated at $1950(95 \%$ HPD $=[1938,1961])$. Clade C-H2 consists mainly of human MSSA,

137 clade C-L consists mainly of livestock MSSA (but also contains 2 MRSA from farms in this study), and clade

138 C-H3 consists of another clade of human MSSA (Clade C-H3-1) from which emerges with the MRCA 139 estimated at 2002, $(95 \%$ HPD $=[1999,2005])$ the clade of human hospital MRSA (Clade C-HM). An additional 140 human MSSA Chinese clade (Clade C-H3-2) shares a MRCA with the 'livestock' clade dated at 1956 $141 \quad(95 \% \mathrm{HPD}=[1946,1966])$.

143 The pig farm CC9 MRSA may be of importance to public health

144 The phylogenetic tree of CC9 S. aureus from this study and previous studies roughly separates into three CC9 145 populations (Figure 3). Towards the root of the tree there is a clade of genomes that possess human immune 146 evasion cluster (IEC) genes ( $c h p, s c n$ and sak) that includes two human clinical MRSA isolates from Chinese

147 Taiwan with a SCCmec V variant. The vast majority of Chinese isolates form a "China pig-farm" clade, are 148 mostly of animal origin, and lack the IEC genes. The China pig-farm clade shares a MRCA with a North 149 American/European clade (the third population which includes an MRSA lineage with a type IVb SCCmec. The

150 China pig-farm clade isolates are almost all MRSA with SCCmec XII and this clade contains all isolates from this study (145 included in this tree). In this clade, of the 45 isolates from other studies, nine were from human

152 clinical cases. As the absence of human IEC genes and the possession of the farm associated antimicrobial resistance genes (ARGs) (tetL, fexA and aac6-Aph2) is a consistent feature in this clade it seems likely that the

154 human clinical isolates probably originated from livestock as it is clear this lineage is found in human carriage samples including farm workers. Notably, there was a single isolate with the human IEC genes isolated from a

156 bacteraemia in a patient from a hospital in Hangzhou (East China) ${ }^{23}$. Within the phylogeny this isolate is surrounded by pig farm isolates from this study.

Different patterns of antimicrobial resistance and virulence genes from pig farms and hospitals

160 An examination of ARGs and selected virulence factors (VFs) in S. aureus sequence data showed that 3 ARGs were frequently found in pig farm isolates (PFA-ARGs), aac6-Aph2 (aminoglycosides ARG) ${ }^{24}$, fexA (phenicols

$162 \mathrm{ARG})^{25}$ and tetL (tetracyclines $\left.\mathrm{ARG}\right)^{26}$; and $3 \mathrm{VFs}$ frequently found in hospital isolates (HA-VFs), scn 163 (Staphylococcal complement inhibitor) ${ }^{27}$, sak (staphylokinase) ${ }^{27}$ and $s p r D$ (small pathogenicity island RNAs) ${ }^{28}$

164 (Figure S2). The PFA-ARG aac6-Aph2 was present in 9/10 clonal lineages of the pig farm MRSA population 
166 particularly in ST239 (41/42) and ST5 (11/16). The ARGs, fexA and tetL, were not present in hospital MRSA isolates (Figure 4). The HA-VF sak was found in 76\% (150/198) of hospital MRSA isolates accounting for

168 20/24 clonal lineages while 82\% (162/198) harboured scn and 83\% (164/198) harboured sprD. The HA-VFs were only present in ST1 and ST59 pig farm MRSA isolates, mostly from farm worker samples. In comparison to ST398 MRSA isolates from patients, ST398 isolates from pig and farm worker samples possessed ARGs aac6-Aph2 and tetL but lacked HA-VFs. The single pig ST1 MRSA isolate in the collection harboured PFA-

172 ARGs aac6-Aph2 which was not present in the hospital ST1 MRSA isolates. Except that a couple of (2/66)

173 hospital ST59 MRSA isolates carried the aac6-Aph2, no other PFA-ARG was detected in ST59 MRSA isolates.

174 HA-VF sak was not detected in pig ST59 MRSA isolate nor in 11/66 hospital ST59 MRSA isolates (Figure 4).

177 An examination of the genomic context of the PFA-ARGs and HA-VFs is illustrated in Figure 5A.

178 A transposon, Tn558, harbouring fexA was detected in the pig farm MSSA ST398 isolate, but not in the pig farm MRSA ST398 isolates or the hospital ST398 isolates. Tn558 could be found in all (313/313) of the fexA positive isolates isolated for this study.

A putative genetic resistance island, GI-PF1, carrying the ARGs tetL and aac6-Aph2 as well as the erythromycin resistance gene ermB, appeared to be present in all the pig farm ST398 isolates, but not in any hospital isolates. The ST1 MRSA isolate from the pig sample carried copies of aac6-Aph2 as well as ermB, which was located on

184 a plasmid (Figure 5B). A part of this plasmid which included the aac6-Aph2 and ermB genes was identical to part of GI-PF1, suggesting a shared origin. The shared region (region 2, Figure 5A) contains ermB and aac6-

186 Aph2, different kinds of transposases such as the Tn3 family transposase and transposase IS1216 as well as the putative transposon Tn552 DNA-invertase bin3. One side of the shared region (region 1) contains genes of a tetracycline efflux MFS transporter (tetL), a plasmid recombination/mobilization protein (pre/mob) and a plasmid replication protein $($ rep $U)$. The remaining part of the island, region 3 , has the cadmium resistance cadDX operon and the cst operon functioning as hydrogen sulfide detoxification. Besides the IS1182 family transposase genes, DNA-invertase gene hin and Tn552 DNA-invertase gene bin3 were also detected in region 3.

192 GI-PF1 region 1 could be detected in $98 \%$ (312/318) of tetL positive isolates. The distribution of GI-PF1 in the 193 ST398 phylogeny is included in Figure 1.

194 The HA-VFs sak, sprD and $s c n$ as well as the virulence gene $\operatorname{chp}$ (chemotaxis inhibitory protein), were located in a prophage region designated phiH1, which has a high level of identity with the $\varphi 3$ phage, Staphylococcus 
196 phage 23MRA (KJ452292.1, 99.43\% identity, 89\% coverage). The phiH1 prophage could be detected in 54\%

197 (144/268) of hospital isolates, but not in pig farm isolates other than the five ST1281 isolates (CC20, all MSSA,

198 one from a farm worker and four from pigs on the same farm).

199 The MRSA ST398 isolates from pig farms and hospitals had different SCCmec elements which are labelled PF-

200 SCC and H-SCC respectively are illustrated in Figure 6. The H-SCC had high similarity with the associated 201 portions of the type V-VT SCCmec of the CA-MRSA ST59 JCSC7190 isolate from Chinese Taiwan (98.89\% 202 identity) and the type Vc SCCmec of the LA-MRSA ST398 reference strain S0385 (98.83\% identity). In 203 comparison to the previously reported similar SCCmec elements, the PF-SCC appeared to have resulted from a 204 homologous recombination event between the type-IX SCCmec of a ST398-t034 MRSA JCSC6943 (Genbank: AB505628.1) from Thailand, and a ST398-t034 MRSA RIVM3897 (Genbank: CP013621.1) from the Netherlands. PF-SCC was detected in all three ST398 MRSA isolated from pig farms. The J1 region of PF-SCC had the genes for the type I restriction and modification system, $h s d R$, $h s d S$, and $h s d M$, while its $\mathrm{J} 2 \mathrm{region}$ had the $c a d D X$ operon, arsRBC operon and the copper-transporting ATPase gene copB.

\section{Discussion}

211 The striking finding from this study was the discovery that MRSA were present in such a high proportion of pig

212 farms while at the same time there was no evidence of shared populations of MRSA in human and animal hosts.

213 This provides evidence for the potential for these farms to be a source of antibiotic resistant $S$. aureus for human

214 infection in China as is the case for LA-MRSA in Europe but no evidence that LA-MRSA are being found in the

215 human healthcare system in China. In Europe, other than a few countries with low MRSA like Norway and

216 Ireland, LA-MRSA are well established in pig farms with farm level prevalence rates between $25.4 \%$ and

$217100.0 \%^{29,30,31}$. These rates are comparable with the MRSA prevalence found in this study although they result 218 from ST398 rather than CC9. Lower rates of CC9 MRSA have been seen in previous studies of Chinese pig 219 farms and slaughterhouse workers ${ }^{18,32}$. While ST398 was found on 3 Chinese pig farms, it was only well 220 established on a single farm as a MSSA lineage. All the ST398 isolates from this study belong to a lineage 221 distinct to the LA-MRSA lineage prevalent in the Europe. Interestingly, this ST398 lineage has been previously 222 been found in China (Figure 1) ${ }^{19}$ but doesn't appear to have become established. In comparison, CC9 was the 223 dominant S. aureus lineage and appears well established in the majority of pig farms from this study, suggesting 224 that CC9 has a competitive advantage over ST398 in the Chinese pig farm environment. CC9 MSSA isolates are not uncommon in European livestock with small numbers of CC9 MRSA reported ${ }^{29}$, suggesting that the 
reverse is true there. Further investigations into those differences may provide useful insights into the factors driving host species adaptation which enable LA-MRSA to become established in pig herds.

228 It is not surprising that out of 42 farm workers carrying MRSA all but 4 carried CC9 MRSA. As is seen in ST398 LA-MRSA, this carriage is likely to be transient, but it does demonstrate how these CC9 MRSA can easily spread from farms to the wider human population. It was therefore surprising not to find any CC9 MRSA among the MRSA isolates collected from the 4 collaborating hospitals in the same region as the farms. It should

232 be noted that it is possible that a larger, more rigorous, or more systematic sampling framework might have 233 found more hospital isolates that might have provided evidence of overlapping populations, in other words, 234 'absence of evidence' is not the same as the 'evidence of absence'.

235 Both MRSA and MSSA ST398 were found in the farms and among the hospital isolates which on the basis of 236 MLST suggested that there may have been movement of this lineage between the animal and human 237 populations. The phylogeny of the ST398 isolates in this study together with the wider collection of ST398 S. 238 aureus genomes revealed that the hospital ST398 MRSA (C-HM) comprised a separate population to those 239 found on the pig farms (C-L) (Figures 1, and 2). The clade C-HM is widely distributed throughout China 240 including isolates from Central, Eastern and Southern regions of the country. The pig farm ST398 MRSA 241 isolates fall within the diversity of the clade C-L consisting mainly of other livestock isolates from China, 242 predominantly MSSA. The livestock ST398 MSSA came from a single pig farm and were also part of this clade.

243 The hospital ST398 MSSA fall in the diversity of another separate clade. The conclusion from this phylogenetic 244 study is that the animal and human populations of ST398 S. aureus in China belong to distinct lineages and 245 there is no evidence that either of the MRSA lineages found on farms is contributing to human MRSA carriage 246 or infection

247 The time-scaled phylogeny of an international collection of ST398 isolates (Figure 2) provides a global context 248 revealing the likely evolution of this sequence type. Previous studies had indicated that the prevalent LA-MRSA 249 ST398 lineage had arisen from a human adapted MSSA, become livestock adapted, acquired SCCmec and 250 became established on livestock farms, particularly in pig herds ${ }^{8}$. This study shows that the prevalent LA251 MRSA shares a common ancestor with a Chinese human MSSA clade (Clade C-H3-2), and that these clades 252 share a common ancestor with the human MSSA clade (Clade C-H3-1) that gave rise to the ST398 MRSA clade 253 (Clade C-HM) from which the hospital isolates from this study came. This topology revealed the diversity of 254 ST398 lineage in Chinese human community and farm animals, highlighting the concern of the emergence of new prevalent CC398 lineages. Given that East Asia was the part of the world where the extant ancestors of the 

evolution of this bacterial species.

258 The investigation of ARGs and VFs identified genes that characterised the MRSA found on farms and in human hospitals. As other studies have found, the human immune evasion cluster of genes found on the $\varphi 3$ phage appear to associate strongly with the source of the isolate being human (238/268 hospital isolates carried at least one of the genes). Similarly, the ARGs tetL, fexA and aac6-Aph2 were strongly associated with a farm origin

262 (322/335 pig farm isolates carried at least one of the genes). Part of this may be a simple consequence of clonal expansion from different founder populations but the identification of a genomic resistance island region containing aac6-Aph2 and ermB in most farm isolates which is identical to a region of a plasmid carried by a ST1 MRSA isolate from a pig shows how readily ARGs can move within S. aureus populations. The consistency and persistence of both the PF-ARGs and the HA-VFs also indicate a continued selection pressure for the retention of these genes. Examination of the SCCmec elements also revealed some consistent differences between the human and pig farm ST398 MRSAs. The SCC found in the hospital isolates (the H-SCC in Figure 6) was a truncated version of a variant of a Type V SCCmec. The source could have been type V-VT (sequence from a ST59 CA-MRSA isolated from Chinese Taiwan) or type Vc (5C2\&5) (sequence from an ST398 LAMRSA isolated from the Netherlands). The truncation results in a length of the SCCmec which is typical of

272 SCCmec types that are associated with CA- MRSA ${ }^{21,33}$. The SCCmec found in the farm ST398 MRSA may have resulted from a recombination of ST398 associated SCCmec type IX and one found in the isolate RIVM3897 (a

274 human ST398 isolate from the Netherlands). These distinctive SCCmec types may provide useful population markers as they currently seem to be restricted to two Chinese clades at present.

276 In conclusion, the presence of such high levels of MRSA isolates on pig farms is of considerable concern. The most likely driver of this is the overuse of antimicrobials in livestock which has been identified as an issue in

278 China ${ }^{34,35}$. Fortunately, this study found no evidence that MRSA from pig farms were contributing to the 279 burden of human MRSA found in samples submitted to clinical microbiology laboratories and while ST398

280 MRSA were found in farms and hospitals there was no shared population that would indicate that transmission 281 had occurred.

\section{Methods}

\section{Sample collection}



each pig farm, nasal swabs of sows, fattening pigs and farm workers, as well as dust samples were collected. If there were sufficient pigs available on each pig farm, at least 6 sows and 20 fattening pigs were randomly selected and sampled (all the pigs on the farm were sampled if numbers were lower than this). All consenting farm workers were sampled anonymously. Nasal swabs were collected using a transport medium (COPAN

290 Diagnostics Inc.). Environmental dust samples were collected using the following method. Three or four sterile tubes containing $10 \mathrm{ml}$ sterilized Mueller Hinton broth (Oxoid, Ltd.) supplemented with $6.5 \% \mathrm{NaCl}$ were opened and exposed in the environment of each selected pig house for at least 10 mins before resealed.

293 Two city hospitals and two county hospitals in the same region were included in this study. The collaborating hospitals retained all clinical MRSA isolates (for the county hospital H3, MSSA isolates were also retained) that had been identified in the course of normal clinical diagnostics during the same period as the pig farm sampling. The sampling of animals and people complied with protocols subject to ethical review by Huazhong Agricultural University (for pig sampling: HZAUSW-2016-013; for human sampling: HZAUHU-2016-006).

\section{S. aureus isolation and identification}

299 All nasal swabs and dust samples were cultured in Mueller Hinton broth with $6.5 \% \mathrm{NaCl}$, at $37^{\circ} \mathrm{C}$ for $16 \mathrm{~h}$ with

300 shaking. For each culture, a full loop of the culture was spread onto MRSA selective plate (CHROMagar

301 Chromogenic Media) and incubated at $35^{\circ} \mathrm{C}$ for $24 \mathrm{~h}$. Putative MRSA colonies on the selective plate appeared to be pink coloured. For each sample, up to 3 pink colonies were selected for further isolation and identification.

303 The MRSA candidate isolates were biochemically profiled using the Phoenix 100 ID/AST System (BD

304 Biosciences); those with positive results were stored as MRSA isolates. All the identified S. aureus isolates were

305 further sequenced. The species and AST of clinical S. aureus isolates obtained from hospitals were also checked using the Phoenix 100 ID/AST System.

\section{Genome sequencing, assembly and annotation}

308 Genomic DNA was prepared from all the isolates grown overnight at $37^{\circ} \mathrm{C}$ in tryptone soy broth (BD 309 Biosciences), using bacterial DNA kits (Omega Bio-Tek). All genomic DNA samples were qualified with an 310 optical density at $260 \mathrm{~nm}\left(\mathrm{OD}_{260 \mathrm{~nm}}\right) / \mathrm{OD}_{280 \mathrm{~nm}}$ ratio between 1.8 and 2 (NanoDrop; Thermo Scientific). Genomic 311 DNA (typically $500 \mathrm{ng}$ ) was used to prepare multiplexed libraries for sequencing on Illumina HiSeq 2000 312 instruments operated according to the manufacturer's instructions with 100 cycle paired-end runs. For all the 313 sequenced genomes, Fastq_screen ${ }^{36}$ was used to map the raw reads to the most common laboratory contaminants to check for contamination as well as published genomes of $S$. aureus to confirm that all isolates 
315 are identified as S. aureus. Genome assemblies of all the isolates were generated using SPAdes $3.11 .1^{37}$.

316 Assemblies with an N50 less than 15,000 were discarded. For assembled data, CheckM (version 1.0 .13$)^{38}$ was

317 used to analyse the contamination of assembled genomes, the genomes with higher than $1.0 \%$ contaminated and

$3180 \%$ strain heterogeneity were excluded. For the six isolates selected for complete genome sequencing, the

319 hospital isolates JL42, JL28 as well as the pig farm isolates 0213-M-4A, 0610-H-2A and 0316-H-5A were

320 further sequenced with Nanopore instruments, while the hospital isolate S82 was sequenced with Pacbio

321 instruments. The sequencing procedures were carried out following the manufacturer's instructions.

322 Unicycler $(0.4 .8)^{39}$ was used for complete genome assembly, the Illumina sequencing data were used as short

323 reads files and the Nanopore (Pacbio) sequencing data were used as long read files. For the parameters used in

324 Unicycler, the Bridging mode chosen was Normal, which means moderate contig size and mis-assembly rate,

325 and the expected linear sequence number was set to 0.

326 For all assembled genomes, Prokka (version 1.12) ${ }^{40}$ was used for genome annotation.

\section{Antimicrobial resistance genes identification}

328 The antimicrobial resistance gene (ARG) database ARG-ANNOT (version 4.0) ${ }^{41}$ was used for ARGs screening.

329 BLAST $+{ }^{42}$ was used for mapping with the annotated genomes for the identification of ARGs. Sequences with a

330 homology of $90 \%$ and an alignment length of $80 \%$ of the corresponding reference gene were considered alleles.

\section{Virulence factors and host-specific genes identification}

332 A database of 216 reported virulence factors (VFs), including virulence, host-specific and other associated genes

333 was created using published research (Table S3). For the sequences mapped to the genes in the database with

334 BLAST+, homology of $90 \%$ and an alignment length of $80 \%$ were used as cut off value for identification.

\section{Determination of the pig farm and hospital associated VFs or ARGs}

336 All the S. aureus isolates, both MRSA and MSSA, were included in the analysis. Based on the distribution of

337 the VFs or ARGs in the pig farm and hospital S. aureus isolates, if an ARG or VF was detected in more than

$33870 \%$ clonal lineages of the pig farm collection and in less than $30 \%$ clonal lineages of the hospital collection, as

339 well as in more than $70 \%$ isolates of pig farm collection and in less than $30 \%$ isolates of hospital collection, the

340 ARG was named as pig farm associated ARG (PFA-ARG) or pig farm associated VF (PFA-VF), conversely, it

341 was named as hospital associated ARG (HA-ARG) or hospital associated VF (HA-VF).

342 Multilocus sequence typing 
343 A database including all published alleles of the seven housekeeping gene fragments for S. aureus multilocus

344 sequence typing (MLST) downloaded from [https://pubmlst.org/] was built to run the BLAST+ process. BLAST

345 results yielding $100 \%$ homology were treated as the same alleles.

346 Isolates selected for phylogenetic analysis

347 A total of 10,535 assembled S. aureus isolates were downloaded from the NCBI genome database (date up to 348 Sep. 2019), MLST analysis was used to identify the clonal lineage of the isolates, all ST398 (for Chinese 349 isolates, CC398 were all included) isolates were selected. 77 Chinese CC398 isolates from a previously 350 published ${ }^{21}$ were additionally included. The data was downloaded from the NCBI SRA database, and was then assembled using SPAdes 3.11.177. Combined with the ST398 isolates from this study, a final collection consisted of 904 CC398 isolates. For all these CC398 isolates, CheckM was used to evaluate the assembly genomes, the genomes with higher than $1.0 \%$ contaminated and $0 \%$ strain heterogeneity (222 isolates) were excluded. According to the BioSample record of each ST398 isolate, 100 isolates with missing location or host information were also excluded. All 115 Chinese CC398 isolates (this study $=36$, NCBI genome database $=37$, NCBI SRA database = 42) were used for Chinese ST398 phylogenetic analysis. The isolates without the collection date record were also excluded, leaving 582 ST398 isolates (this study $=35$, NCBI genome database $=505$, NCBI SRA database $=42)$ as the international ST398 collection $($ Table S2).

\section{Phylogenetic analysis of the Chinese CC398 isolates}

360 Snippy (version 4.4.5) [https://github.com/tseemann/snippy] was used to do the genome alignment of the 110

361 Chinese ST398 isolates. The pig-farm ST398 MRSA in this study 0213-M-4A was used as the reference. Then,

362 for the alignment file, a recombination-removal tool Gubbins (version 2.3 .5$)^{43}$ was used to predict the recombination regions. All the recombination regions were marked as "N", and for the alignment results, all the sites with more than one "N" or gap were trimmed off. For the filtered alignment result, RAxML-NG (version 0.9.0) ${ }^{44}$ was used to build the phylogenetic tree with 500 times bootstrap. A MRSA ST36 strain $^{8}\left(\right.$ GenBank: $^{2}$ BX571856.1) as well as a MRSA ST45 strain (GenBank: CP006044.1) were used as outgroups respectively to double check the root of the tree.

\section{BEAST analysis of international ST398 isolates}

369 To determine the international phylogeny and molecular clock of ST398 isolates, BEAST 1.8.2 $2^{22}$ was used to 370 perform the calculation. For the international ST398 collection $(\mathrm{n}=582)$, a RAxML-NG tree was built 371 following the pipeline as described above. Based on the phylogenetic tree, all the clades whose average branch length distance was below 0.00005 were collapsed. Each of the collapsed, as well as the un-collapsed, clades 
374 location information was the same, only one of the isolates was selected. Thus, 208 isolates (this study $=11$,

375 NCBI genome database $=168$, NCBI SRA database $=29)$ were selected for the analysis with BEAST. Temporal signal of the 208 isolates was investigated using R script provided in the Murray et al., $(2015)^{45}$. The results showed the positive correlation between genetic divergence and sampling time. For the alignment file of the 208 isolates (0213-M-4A was the reference), the recombination regions were predicted with Gubbins and then masked as "N", and all the sites containing "N" were removed. To account for different evolutionary processes acting at synonymous, non-synonymous, RNA, and non-coding sites ${ }^{46}$, the evolutionary model was partitioned into first and second, third, non-coding and RNA sites according to the reference strain 0213-M-4A. SNPs for each group and the number of the invariant nucleotide sites in each group were calculated, and the information was included in the analysis. For all partitions, we used an $\mathrm{HKY}+\Gamma$ substitution model with the uncorrelated lognormal relaxed clock model.

\section{Phylogenetic analysis of the Chinese ST9 isolates}

386 For clarity, the isolate number from the study was reduced - for isolates from the same pig farm and same host, 387 that have the same ARGs and VFs patterns and with less than 30 SNPs, only one isolate was selected. The CC9 isolate 0213-P-3B with complete genomic sequence was used as the reference genome and a MRSA ST5 strain (GenBank: NC_002745.2) as well as a ST97 isolate NCTC10344 (GenBank: LS483324.1) (not show in figure) were used as outgroups respectively to root the tree. The other approaches were the same as the phylogenetic analysis of the Chinese CC398 isolates that mentioned above.

\section{The analysis of canSNP}

393 The presence of three canonical SNPs (canSNP) was identified for each ST398 genome as described by Stegger

394 et al., (2013) ${ }^{10}$ to determine if isolates were members of the human or livestock clades of LA-MRSA.

\section{SCCmec identification}

396 The reference sequences of each reported SCCmec elements from I to XIV were collected as a database for 397 SCCmec identification (Table S4). The mapping region with the identity higher than $80 \%$ in different contigs of 398 a genome sequence were accumulated to calculate the SCCmec type coverage. For each isolate, from all the 399 mapped reference SCCmec elements with higher than $70 \%$ overall coverage, the one with the highest coverage 400 was identified as the SCCmec type of the isolate. The SCCmec typing results were double checked with the 401 SCCmecFinder ${ }^{47}$, the results of the two methods were consistent. 
bioRxiv preprint doi: https://doi.org/10.1101/2021.08.02.454852; this version posted August 3, 2021. The copyright holder for this preprint (which was not certified by peer review) is the author/funder. All rights reserved. No reuse allowed without permission.

Other genomic elements of the six ST398 isolates with complete genomic sequence were analysed to look for

prophages and islands. PHASTER ${ }^{48}$ was used to predict any prophage region, while IslandViewer $4{ }^{49}$ was used

to detect any genomic island region. Searches for genomic elements including prophages, genomic islands,

plasmids, and transposons among the whole S. aureus collection in this study was performed using BLAST to

map regions of interest to the genome sequence of each isolate using an $80 \%$ threshold for identity.

\section{Accession numbers}

All the $S$. aureus genomes sequenced in this study are available under the NCBI BioProject: PRJNA660925.

\section{Acknowledgements}

412 This work was funded by the National Natural Science Foundation of China (NSFC) grant 81661138003 (to R.

Z.), and the UK Medical Research Council grant MR/P007201/1 (to M.A.H., A.J.G. and A. W. T.).

\section{Reference}

416 1. Lakhundi, S. \& Zhang, K. Methicillin-Resistant Staphylococcus aureus: Molecular Characterization, Evolution, and Epidemiology. Clinical microbiology reviews vol. 31 1-103 (2018).

419

420

421

422

423

424

425

426

427

428

429

430

431

432

433

434

435

436

437

438

439

440

441

442

443

2. García-Álvarez, L. et al. Meticillin-resistant Staphylococcus aureus with a novel mecA homologue in human and bovine populations in the UK and Denmark: A descriptive study. Lancet Infect. Dis. 11, 595-603 (2011).

3. Aires-de-Sousa, M. Methicillin-resistant Staphylococcus aureus among animals: current overview. Clin. Microbiol. Infect. 23, 373-380 (2017).

4. Voss, A., Loeffen, F., Bakker, J., Klaassen, C. \& Wulf, M. Methicillin-resistant Staphylococcus aureus in Pig Farming. Emerg. Infect. Dis. 11, 1965-1966 (2005).

5. van Cleef, B. A. G. L. et al. Livestockassociated methicillin- resistant staphylococcus aureus in humans, Europe. Emerg. Infect. Dis. 17, 502-505 (2011).

6. Smith, T. C. et al. Methicillin-resistant Staphylococcus aureus (MRSA) strain ST398 is present in midwestern U.S. swine and swine workers. PLoS One 4, 1-6 (2009).

7. Birgitte Borck Høg \& Helle Korsgaard. DANMAP - Use of antimicrobial agents and occurrence of antimicrobial resistance in bacteria from food animals, food and humans in Denmark. 17-31 (2016).

8. Price, L. B. et al. Staphylococcus aureus CC398: Host Adaptation and Emergence of Methicillin Resistance in Livestock. MBio 3, 1-7 (2012).

9. Ward, M. J. et al. Time-scaled evolutionary analysis of the transmission and antibiotic resistance dynamics of Staphylococcus aureus clonal complex 398. Appl. Environ. Microbiol. 80, 7275-7282 (2014).

10. Stegger, M. et al. Rapid differentiation between livestock-associated and livestock-independent staphylococcus aureus CC398 clades. PLoS One 8, 1-5 (2013).

11. Zhou, W. et al. WGS analysis of ST9-MRSA-XII isolates from live pigs in China provides insights into transmission among porcine, human and bovine hosts. J. Antimicrob. Chemother. 73, 2652-2661 (2018).

12. Wan, M. T., Lauderdale, T. L. \& Chou, C. C. Characteristics and virulence factors of livestock associated ST9 methicillin-resistant Staphylococcus aureus with a novel recombinant staphylocoagulase type. Vet. Microbiol. 162, 779-784 (2013).

13. Espinosa-Gongora, C. et al. Phenotypes and genotypes of old and contemporary porcine strains indicate a temporal change in the S. aureus population structure in pigs. PLoS One 9, 1-5 (2014).

14. Sun, J., Yang, M., Sreevatsan, S. \& Davies, P. R. Prevalence and Characterization of Staphylococcus aureus in Growing Pigs in the USA. PLoS One 10, 1-14 (2015).

15. Cuny, C., Wieler, L. H. \& Witte, W. Livestock-Associated MRSA: The impact on humans. Antibiotics 4, 521-543 (2015).

16. Matuszewska, M., Murray, G. G. R., Harrison, E. M., Holmes, M. A. \& Weinert, L. A. The Evolutionary Genomics of Host Specificity in Staphylococcus aureus. Trends Microbiol. 28, 465-477 
(2020).

17. Cui, S. et al. Isolation and characterization of methicillin-resistant Staphylococcus aureus from swine and workers in China. J. Antimicrob. Chemother. 64, 680-683 (2009).

18. Li, J. et al. Characterization of pig-associated methicillin-resistant Staphylococcus aureus. Vet. Microbiol. 201, 183-187 (2017).

19. Sun, C. et al. Genomic analysis of Staphylococcus aureus along a pork production chain and in the community, Shandong Province, China. Int. J. Antimicrob. Agents 54, 8-15 (2019).

20. Chen, H. et al. Whole-Genome Analysis of Livestock-Associated Methicillin-Resistant Staphylococcus aureus Sequence Type 398 Strains Isolated From Patients With Bacteremia in China. J. Infect. Dis. 221, S220-S228 (2020).

21. He, L. et al. Detection and analysis of methicillin-resistant human-adapted sequence type 398 allows insight into community-associated methicillin-resistant Staphylococcus aureus evolution. Genome Med. 10, 1-14 (2018).

22. Drummond, A. J., Suchard, M. A., Xie, D. \& Rambaut, A. Bayesian phylogenetics with BEAUti and the BEAST 1.7. Mol. Biol. Evol. 29, 1969-1973 (2012).

23. Jin, Y. et al. Characterization of highly virulent community-associated methicillin-resistant Staphylococcus aureus ST9-SCCmec XII causing bloodstream infection in China. Emerg. Microbes Infect. 9, 2526-2535 (2020).

24. Li, C. R. et al. In vitro antibacterial activity of vertilmicin and its susceptibility to modifications by the recombinant AAC(6')-APH(2") enzyme. Antimicrob. Agents Chemother. 52, 3875-3882 (2008).

25. Kehrenberg, C. \& Schwarz, S. Florfenicol-chloramphenicol exporter gene fexA is part of the novel transposon Tn558. Antimicrob. Agents Chemother. 49, 813-815 (2005).

26. McMurry, L. M., Park, B. H., Burdett, V. \& Levy, S. B. Energy-dependent efflux mediated by class L (TetL) tetracycline resistance determinant from streptococci. Antimicrob. Agents Chemother. 31, 16481650 (1987).

27. Kumagai, R. et al. Quadruple or quintuple conversion of hlb, sak, sea (or sep), scn, and chp genes by bacteriophages in non- $\beta$-hemolysin-producing bovine isolates of Staphylococcus aureus. Vet. Microbiol. 122, 190-195 (2007).

28. Chabelskaya, S., Gaillot, O. \& Felden, B. A Staphylococcus aureus small RNA is required for bacterial virulence and regulates the expression of an immune-evasion molecule. PLoS Pathog. 6, 1-11 (2010).

29. Parisi, A. et al. MRSA in swine, farmers and abattoir workers in Southern Italy. Food Microbiol. 82, 287-293 (2019).

30. The European Union summary report on antimicrobial resistance in zoonotic and indicator bacteria from humans, animals and food in 2015. EFSA J. 15, (2017).

31. The European Union summary report on antimicrobial resistance in zoonotic and indicator bacteria from humans, animals and food in 2017. EFSA J. 17, (2019).

32. Wang, X. L. et al. Phenotypic and molecular characteristics of Staphylococcus aureus and methicillinresistant Staphylococcus aureus in slaughterhouse pig-related workers and control workers in Guangdong Province, China. Epidemiol. Infect. 145, 1843-1851 (2017).

33. Møller, J. K. et al. International travel as source of a hospital outbreak with an unusual meticillinresistant Staphylococcus aureus clonal complex 398, Denmark, 2016. Eurosurveillance 24, (2019).

34. Ying, G. G. et al. China Must Reduce Its Antibiotic Use. Environ. Sci. Technol. 51, 1072-1073 (2017).

35. Hu, Y. \& Cheng, H. Health risk from veterinary antimicrobial use in China's food animal production and its reduction. Environ. Pollut. 219, 993-997 (2016).

36. Wingett, S. W. \& Andrews, S. Fastq screen: A tool for multi-genome mapping and quality control [version 1; referees: 3 approved, 1 approved with reservations]. F1000Research 7, 1-13 (2018).

37. Bankevich, A. et al. SPAdes: A new genome assembly algorithm and its applications to single-cell sequencing. J. Comput. Biol. 19, 455-477 (2012).

38. Parks, D. H., Imelfort, M., Skennerton, C. T., Hugenholtz, P. \& Tyson, G. W. CheckM: Assessing the quality of microbial genomes recovered from isolates, single cells, and metagenomes. Genome Res. 25 , 1043-1055 (2015).

39. Wick, R. R., Judd, L. M., Gorrie, C. L. \& Holt, K. E. Unicycler: Resolving bacterial genome assemblies from short and long sequencing reads. PLoS Comput. Biol. 13, 1-22 (2017).

40. Seemann, T. Prokka: Rapid prokaryotic genome annotation. Bioinformatics 30, 2068-2069 (2014).

41. Gupta, S. K. et al. ARG-annot, a new bioinformatic tool to discover antibiotic resistance genes in bacterial genomes. Antimicrob. Agents Chemother. 58, 212-220 (2014).

42. Camacho, C. et al. BLAST+: Architecture and applications. BMC Bioinformatics 10, 1-9 (2009).

43. Croucher, N. J. et al. Rapid phylogenetic analysis of large samples of recombinant bacterial whole genome sequences using Gubbins. Nucleic Acids Res. 43, e15 (2015).

44. Kozlov, A. M. et al. RAxML-NG: A fast, scalable and user-friendly tool for maximum likelihood 
phylogenetic inference. Bioinformatics 35, 4453-4455 (2019).

45. Murray, G. G. R. et al. The effect of genetic structure on molecular dating and tests for temporal signal. 80-89 (2016) doi:10.1111/2041-210X.12466.

46. Bofkin, L. \& Goldman, N. Variation in evolutionary processes at different codon positions. Mol. Biol. Evol. 24, 513-521 (2007).

47. Kaya, H. et al. SCCmecFinder, a Web-Based Tool for Typing of Staphylococcal Cassette Chromosome mec in Staphylococcus aureus Using Whole-Genome Sequence Data. mSphere 3, 1-9 (2018).

48. Arndt, D. et al. PHASTER: a better, faster version of the PHAST phage search tool. Nucleic Acids Res. 44, W16-W21 (2016).

49. Bertelli, C. et al. IslandViewer 4: Expanded prediction of genomic islands for larger-scale datasets. Nucleic Acids Res. 45, W30-W35 (2017). 


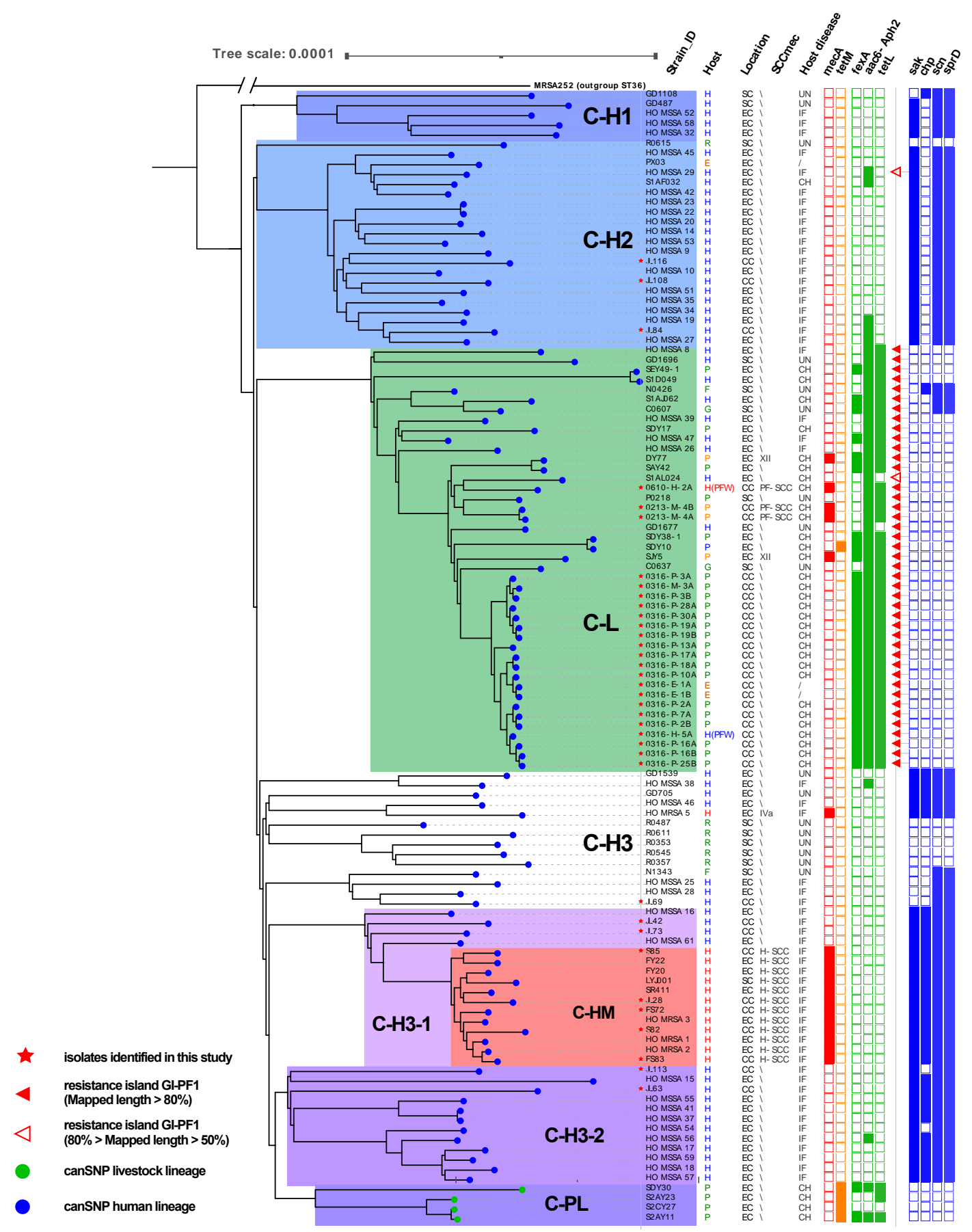

Figure 1. The phylogenetic tree illustrates the relationship of the Chinese CC398 isolates. ST398, 8 other STs) from this study and other published studies is shown. An MRSA ST36 strain (GenBank: BX571856.1) was used as the out group. Location: EC, East China; SC, South China; CC, Central China. Host: B, Bovine; H, Human; P, Porcine; F, Feline; R, Rodent; G, Galline/Chicken; E, Environment; H(PFW), Human from a pig farm worker sample. SCCmec: For each isolate, the SCCmec type was determined if more than $70 \%$ of SCCmec elements could be detected in the genome. Some mecA negative isolates were found to have SCCmec elements using this method. Host disease: UN, Unknown; IF, Infected with S. aureus; CH, Clinically healthy. ARGs and VFs: the filled squares indicate the presence and empty squares the absence of individual genes indicated at the head of each column. 


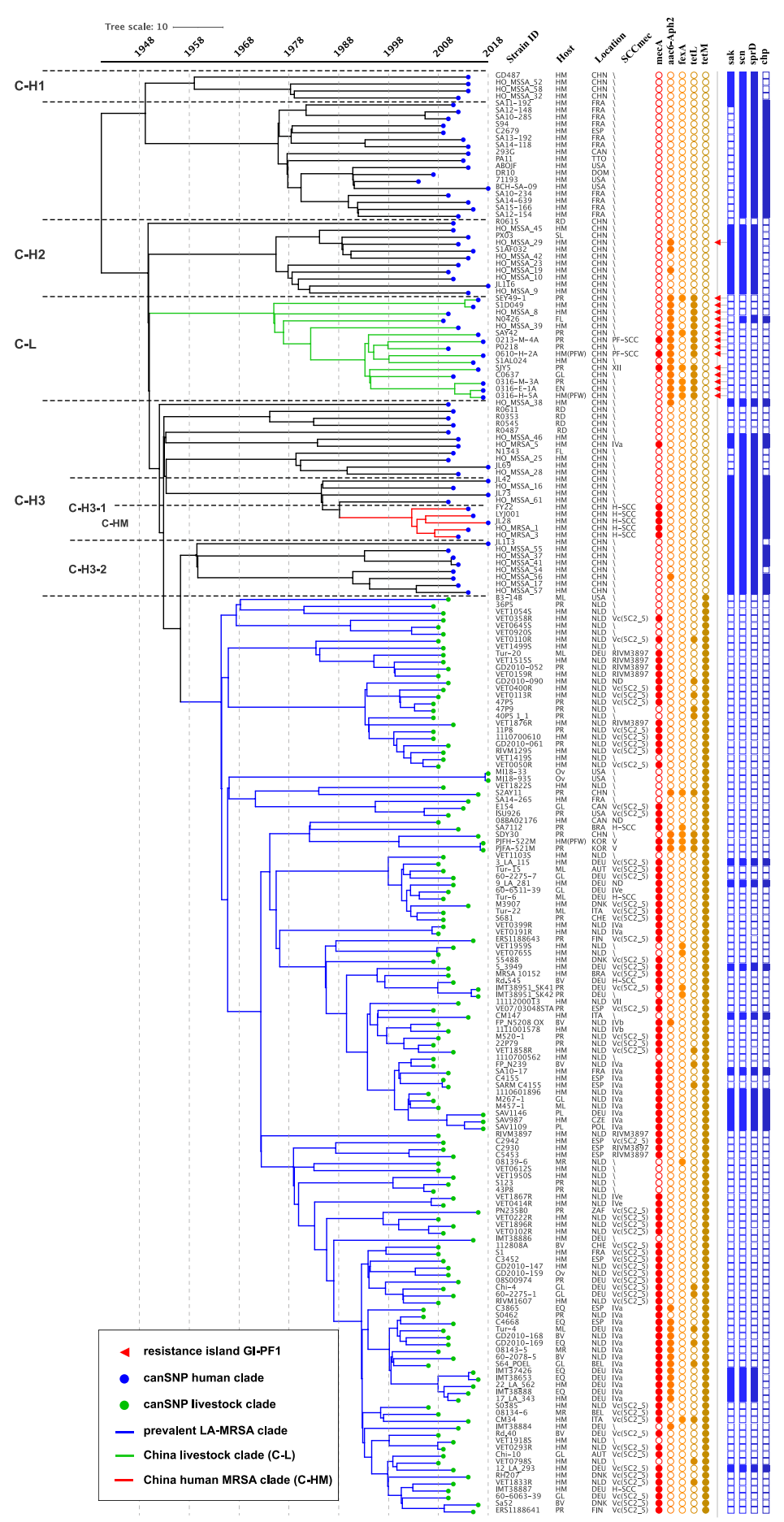

Figure 2. Phylogeny and molecular dating of the international ST398 isolates.

A time-measured phylogenetic tree using an international collection of ST398 isolates was obtained using BEAST2. A total of 207 ST398 isolates (11 from this study, and 196 from the NCBI genome collection) were used in the analysis. Host: HM, Human; PR, Porcine; EN, Environment; HM(PFW), Human from a pig farm worker; MR, Murine; BV, Bovine; GL, Galline; ML, Meleagrine; EQ, Equine; OV, Ovine; FL, Feline; RD, Rodentia; PL, Poultry. Location: DNK, Denmark; USA, United States of America; NLD, Netherlands; CHN, China; BEL, Belgium; CAN, Canada; DEU, Germany; CHE, Switzerland; ESP, Spain; AUT, Austria; ITA, Italy; DMA, Dominica; BRA, Brazil; TTO, Trinidad \& Tobago; FIN, Finland; KOR, South Korea; ZAF, South Africa; FRA, France; POL, Poland; CZE, Czech Republic. SCCmec: For each isolate, the SCCmec type was determined if more than $70 \%$ of SCCmec elements could be detected in the genome. 


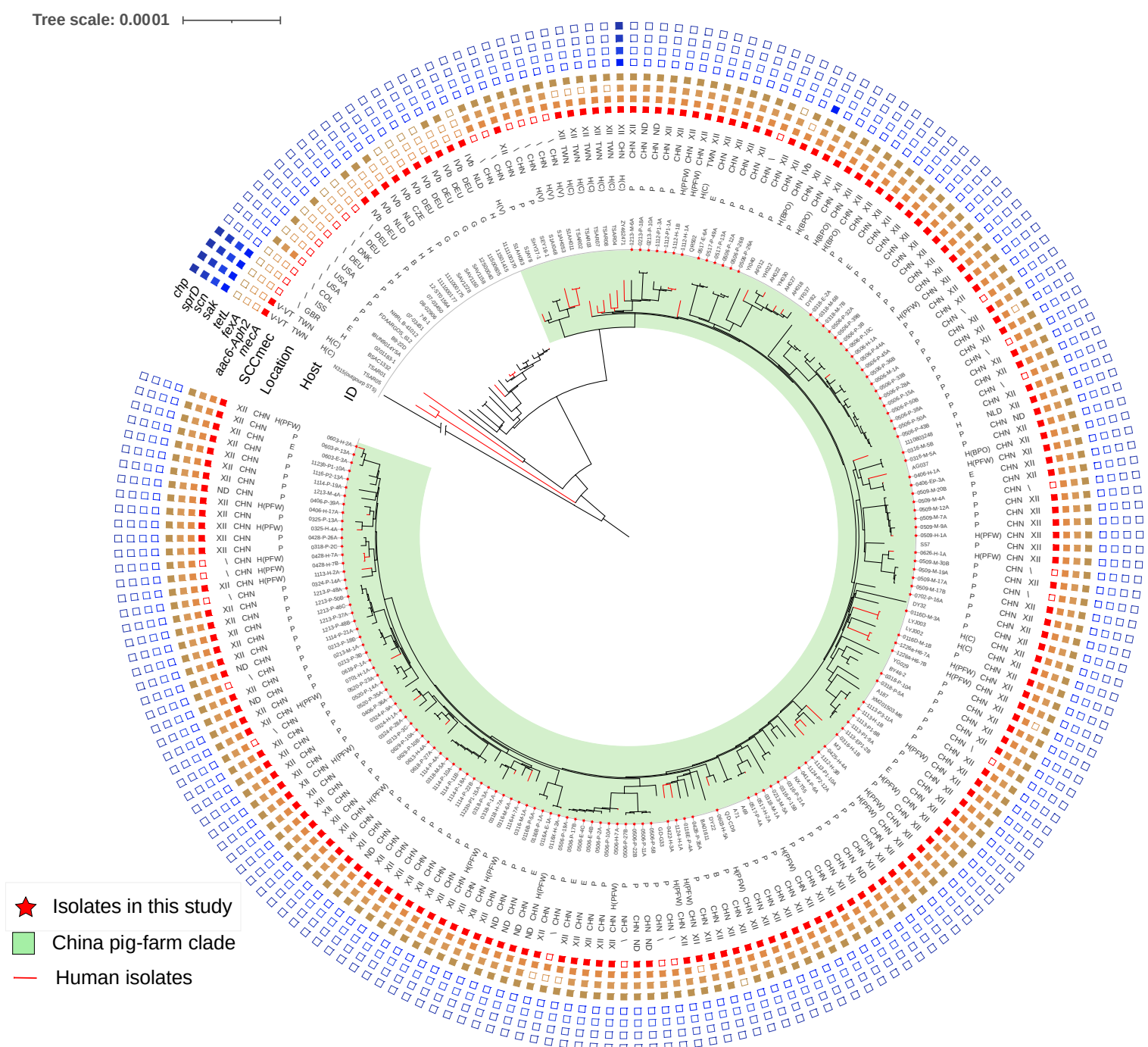

Figure 3. The phylogenetic tree illustrates the relationship of the international ST9 isolates.

Based on whole genome SNPs, a phylogenetic tree consisting of 144 isolates selected from this study and 63 isolates from other reports is shown. A MRSA ST5 strain (GenBank: NC_002745.2) was used as the out group. Location: CHN, China; COL, Colombia; CZE, Czech Republic; DEU, Germany; DNK, Denmark; GBR, United Kingdom; ISS, International Space Station Air (U.S. Lab); NLD, Netherlands; TWN, Chinese Taiwan; USA, United States. Host: B, Bovine; E, Environment; G, Galline/ Poultry; H, Human; H(C), from a human S. aureus infection clinical sample; H(BPO), from a backyard pig farm owner sample; $\mathrm{H}(\mathrm{PFW})$, from a pig farm worker sample; $\mathrm{H}(\mathrm{V})$, from a village community sample. P, Porcine. SCCmec: ND, mecA positive but not detected published SCCmec. ARGs and VFs: the filled squares indicate the presence and empty squares the absence of individual genes indicated at the head of each column. 


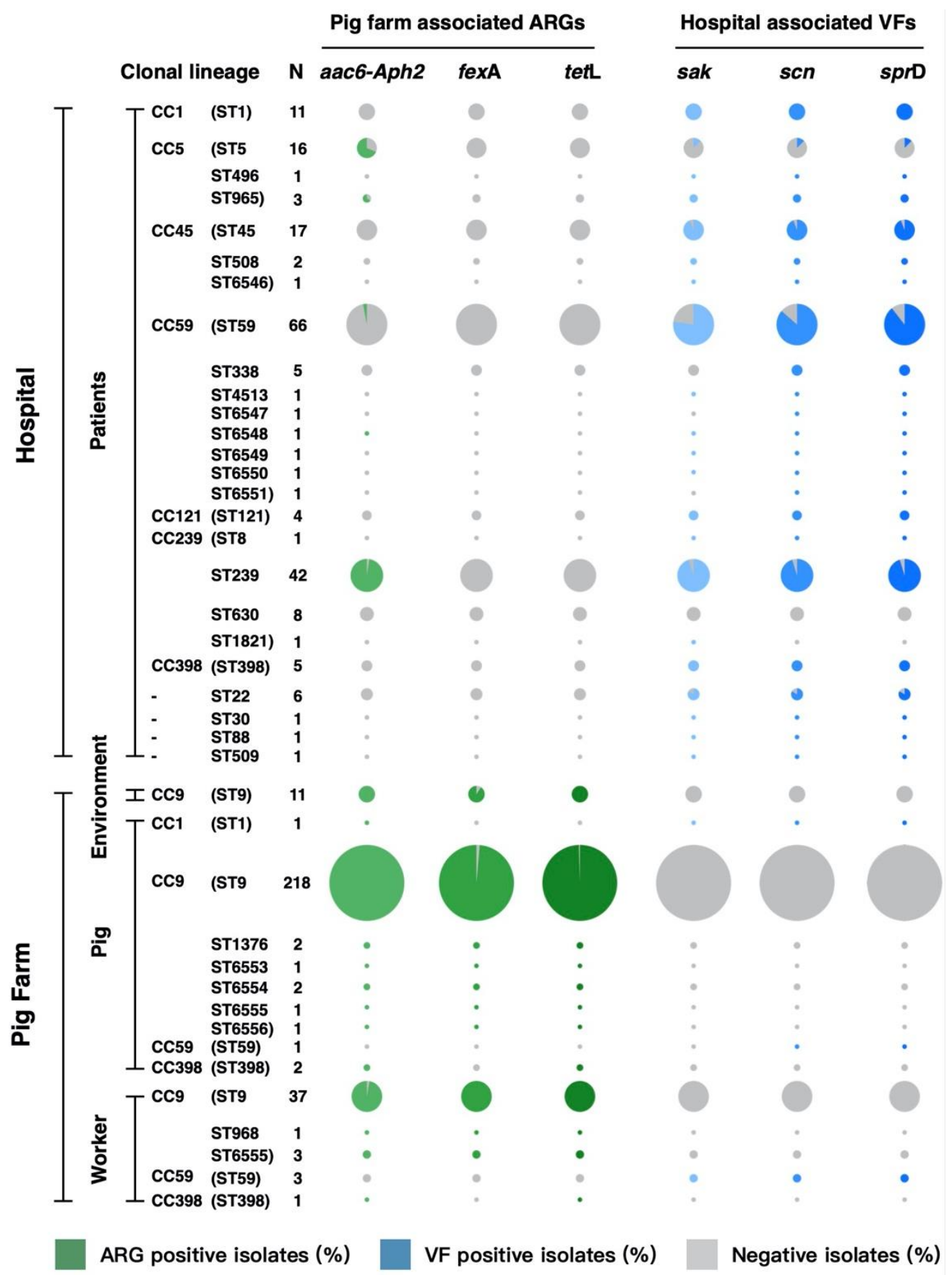

560 Figure 4. Distribution of pig farm associated antimicrobial resistance genes and hospital 561 associated virulence factor genes among clonal lineages.

562 The distribution of three antimicrobial resistance genes (ARGs) and three hospital associated 563 virulence factor genes (VFs) among clonal lineages of all isolates is shown. The pie charts in each 564 row, indicate the proportion of the isolates from each ST harboring individual ARGs or VFs as 565 defined by the column, with the size of each pie chart proportional to the number of isolates. 


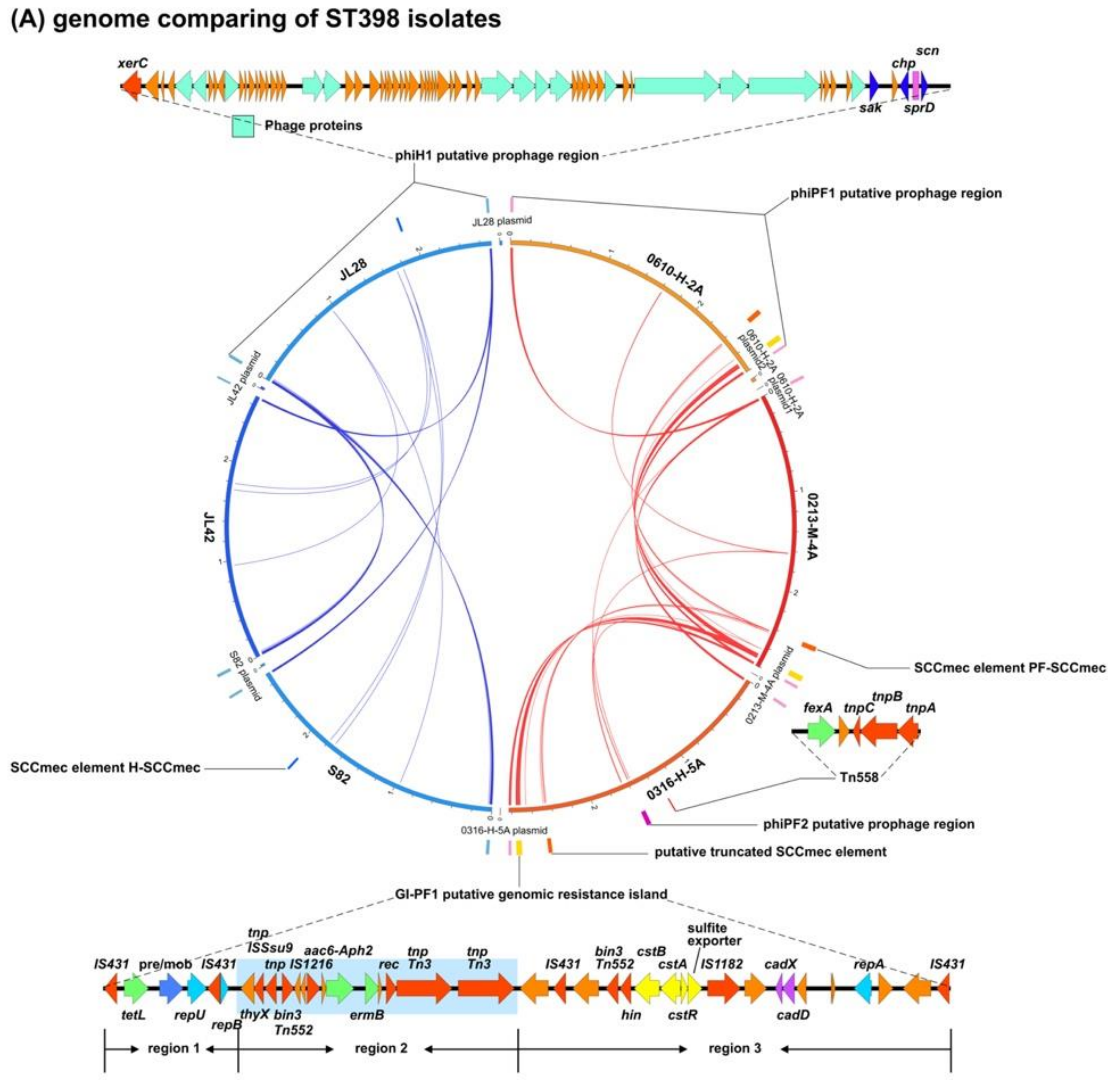

(B) pig farm ST1 MRSA plasmid

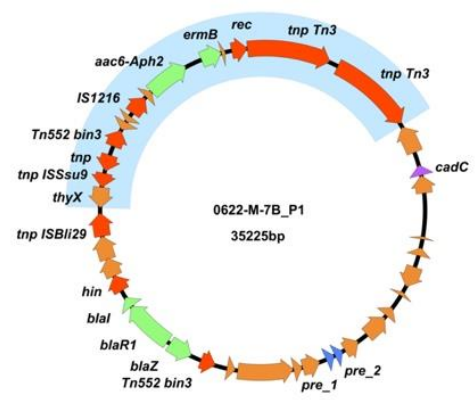

Figure 5. Genomic elements carrying pig farm associated antimicrobial resistance genes and hospital associated virulence factor genes.

The genomic elements harboring the pig farm associated ARGs or hospital associated VFs were identified by comparing the ST398 isolates of pig farms and hospitals. (A) The distribution of the genomic elements in six ST398 completed genomes are shown: JL28, hospital ST398 MSSA isolate; JL42 and S82, hospital ST398 MRSA isolates; 0213-M-4A: pig farm MRSA isolate from a sow nasal sample; 0610-H-2A: pig farm MRSA isolate from a farm worker nasal sample; 0316-H-5A: pig farm MSSA isolate from a farm worker nasal sample. The genomic sequences shared only between the hospital isolates are indicated using blue curves, and genomic sequences shared only between the pig farm isolates are indicated using red curved lines. Diagrammatic representations of the genomic elements are illustrated, and their locations are shown adjacent to each genome included in the central circular figure. Individual transposon associated genes are shaded red, ARGs are shaded green and purple is used to show genes conferring resistance to metals. (B) The panel contains a diagram of a plasmid from the ST1 pig farm isolate 0622-M-7B. When compared to the ST1 MRSA isolates from the hospitals, the pig farm MRSA ST1 isolates had two additional ARGs, aac6-Aph2 and ermB. These two ARGs were found in a plasmid with a sequence that was identical with region 2 of the GI-PF1 identified in the pig farm ST398 isolates (highlighted in blue). 


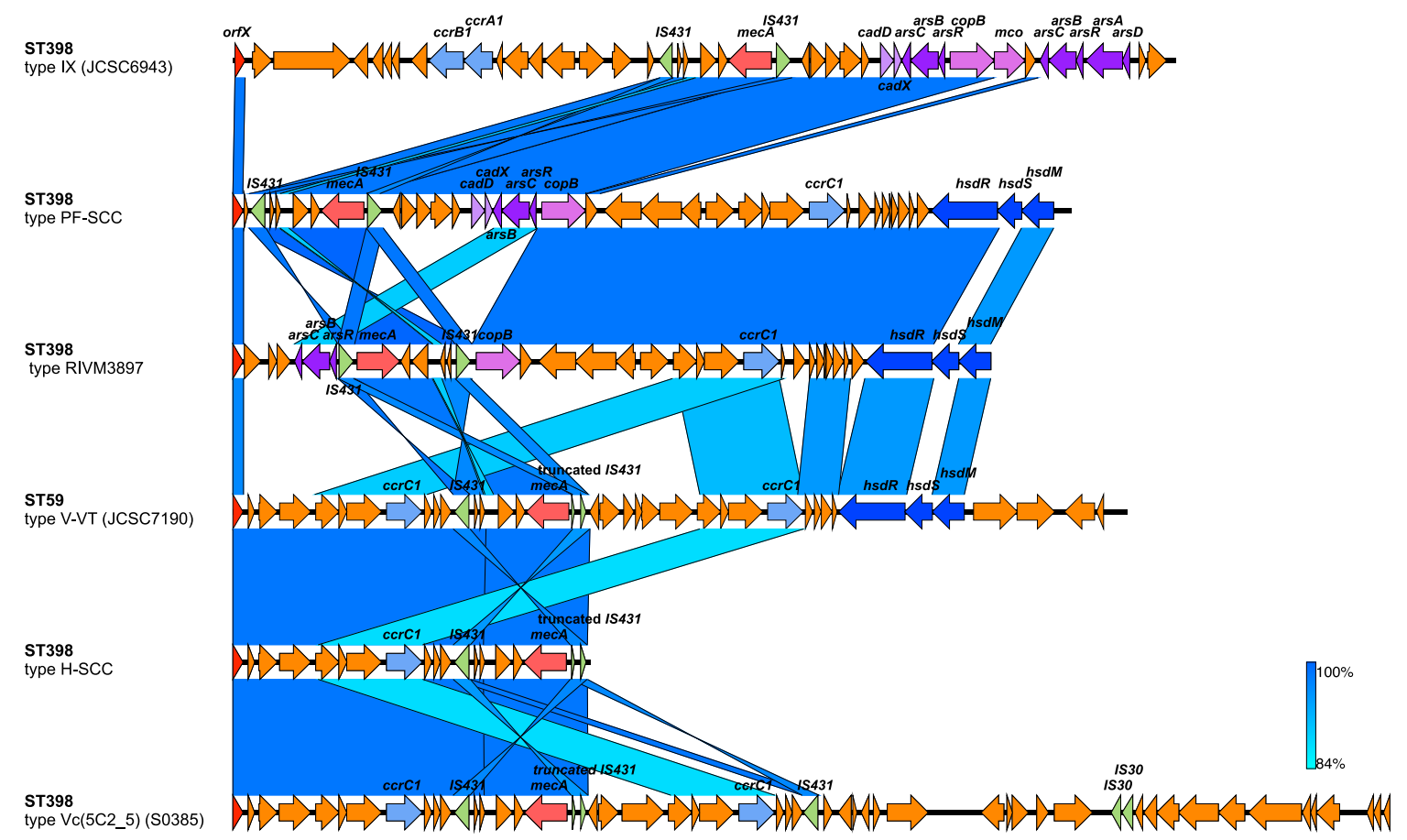

Figure 6. Structures of SCCmec elements identified in ST398 MRSA isolates.

The diagram shows the main genetic elements and regions of similarity between the SCCmec regions found in MRSA in this study with reference SCCmec types. Sources of the SCCmecs were the hospital MRSA ST398 isolate JL28 (H-SCC), and the pig farm MRSA ST398 isolate 0213-M-4A (PF-SCC). Type V SCCmec from ST59 strain JCSC7190 (GenBank: AB512767), type IX SCCmec from ST398 strain JCSC6943 (GenBank: AB505628), the SCCmec from MRSA ST398 strain RIVM3897 (GenBank: CP013621.1; the SCCmec element was determined from 33795 to $69446 \mathrm{bp}$ ) and SCCmec from ST398 strain S0835 (GenBank:AM990992.1; the SCCmec element was determined from 33806 to 88218 bp) are shown for comparison. Regions of similarity ( $85 \%$ to $100 \%$ ) between each pair of elements are linked with blue bars where the intensity of shading illustrating the degree of similarity. 
bioRxiv preprint doi: https://doi.org/10.1101/2021.08.02.454852; this version posted August 3, 2021. The copyright holder for this preprint (which was not certified by peer review) is the author/funder. All rights reserved. No reuse allowed without permission.

Table 1 The distribution of the whole $S$. aureus collection according to different sample sources.

\begin{tabular}{|c|c|c|c|c|c|c|c|c|c|c|}
\hline \multirow{3}{*}{$\begin{array}{l}\text { Clonal } \\
\text { complex }\end{array}$} & \multirow{3}{*}{$\begin{array}{l}\text { Sequence } \\
\text { type }\end{array}$} & \multirow{3}{*}{$\begin{array}{l}\text { SCCmec } \\
\text { type }\end{array}$} & \multicolumn{6}{|c|}{ Pig Farm } & \multicolumn{2}{|c|}{ Hospital } \\
\hline & & & \multicolumn{2}{|c|}{ Farm worker } & \multicolumn{2}{|c|}{ Environment } & \multicolumn{2}{|l|}{ Pig } & \multirow{2}{*}{ MRSA } & \multirow{2}{*}{ MSSA } \\
\hline & & & MRSA & MSSA & MRSA & MSSA & MRSA & MSSA & & \\
\hline \multirow[t]{5}{*}{ CC1 } & ST1 & $\mathrm{IVb}$ & - & - & - & - & - & - & 11 & - \\
\hline & & IVc & - & - & - & - & 1 & - & - & - \\
\hline & & - & - & - & - & - & - & 5 & - & 6 \\
\hline & ST188 & - & - & - & - & - & - & - & - & 16 \\
\hline & ST6544 & - & - & - & - & - & - & - & - & 1 \\
\hline CC5 & ST5 & IIa & - & - & - & - & - & - & 14 & - \\
\hline & & IVa & - & - & - & - & - & - & 2 & - \\
\hline & & - & - & - & - & - & - & 4 & - & 4 \\
\hline & ST6 & - & - & - & - & - & - & - & - & 5 \\
\hline & ST552 & - & - & - & - & - & - & - & - & 1 \\
\hline & ST496 & IIa & - & - & - & - & - & - & 1 & - \\
\hline & ST965 & IVc & - & - & - & - & - & - & 3 & - \\
\hline & ST2114 & - & - & - & - & - & - & - & - & 1 \\
\hline CC9 & ST9 & XII & 35 & - & 9 & - & 195 & - & - & - \\
\hline & & ND & 2 & - & 2 & - & 23 & - & - & - \\
\hline & & - & - & 2 & - & - & - & 14 & - & - \\
\hline & ST6553 & XII & - & - & - & - & 1 & - & - & - \\
\hline & ST6554 & XII & - & - & - & - & 2 & - & - & - \\
\hline & ST6555 & XII & 3 & - & - & - & 1 & - & - & - \\
\hline & ST6556 & XII & - & - & - & - & 1 & - & - & - \\
\hline & ST968 & ND & 1 & - & - & - & - & - & - & - \\
\hline & ST1376 & XII & - & - & - & - & 2 & - & - & - \\
\hline $\mathrm{CC45}$ & ST45 & IVa & - & - & - & - & - & - & 17 & - \\
\hline & ST508 & IVi & - & - & - & - & - & - & 2 & - \\
\hline & & - & - & - & - & - & - & - & - & 1 \\
\hline & ST6546 & IVi & - & - & - & - & - & - & 1 & - \\
\hline CC59 & ST59 & IVa & 3 & - & - & - & - & - & 48 & - \\
\hline & & $\mathrm{IVb}$ & - & - & - & - & - & - & 5 & - \\
\hline & & V-VT & - & - & - & - & 1 & - & 13 & - \\
\hline & ST6547 & IVa & - & - & - & - & - & - & 1 & - \\
\hline & ST6548 & IVa & - & - & - & - & - & - & 1 & - \\
\hline & ST6549 & IVg & - & - & - & - & - & - & 1 & - \\
\hline & ST6550 & IVa & - & - & - & - & - & - & 1 & - \\
\hline & ST6551 & IVa & - & - & - & - & - & - & 1 & - \\
\hline & ST338 & V-VT & - & - & - & - & - & - & 5 & - \\
\hline & ST4513 & IVa & - & - & - & - & - & - & 1 & - \\
\hline CC121 & ST121 & $\mathrm{V}$ & - & - & - & - & - & - & 4 & - \\
\hline & & - & - & - & - & - & - & - & - & 4 \\
\hline & ST6543 & - & - & - & - & - & - & - & - & 1 \\
\hline CC239 & ST8 & IVa & - & - & - & - & - & - & 1 & - \\
\hline & ST239 & III & - & - & - & - & - & - & 42 & - \\
\hline & ST623 & - & - & - & - & - & - & - & - & 1 \\
\hline & ST630 & H-SCC & - & - & - & - & - & - & 2 & - \\
\hline & & $\mathrm{V}$ & - & - & - & - & - & - & 6 & - \\
\hline & & - & - & - & - & - & - & - & - & 2 \\
\hline & ST1821 & V & - & - & - & - & - & - & 1 & - \\
\hline CC398 & ST398 & H-SCC & - & - & - & - & - & - & 5 & - \\
\hline & & PF-SCC & 1 & - & - & - & 2 & - & - & - \\
\hline & & - & - & 1 & - & 2 & - & 17 & - & 7 \\
\hline & ST6545 & - & - & - & - & - & - & - & - & 1 \\
\hline CC1281 & ST1281 & - & - & 1 & - & - & - & 4 & - & 1 \\
\hline & ST2631 & - & - & - & - & - & - & - & - & 1 \\
\hline- & ST7 & - & - & - & - & - & - & - & - & 2 \\
\hline- & ST15 & - & - & - & - & - & - & - & - & 1 \\
\hline- & ST22 & IVa & - & - & - & - & - & - & 1 & - \\
\hline- & & V-VT & - & - & - & - & - & - & 5 & - \\
\hline - & & - & - & - & - & - & - & - & - & 3 \\
\hline- & ST25 & - & - & - & - & - & - & - & - & 8 \\
\hline - & ST30 & IVc & - & - & - & - & - & - & 1 & - \\
\hline - & ST88 & V-VT & - & - & - & - & - & - & 1 & - \\
\hline- & ST509 & IVa & - & - & - & - & - & - & 1 & - \\
\hline - & ST672 & - & - & - & - & - & - & - & - & 1 \\
\hline- & ST944 & - & - & - & - & - & - & - & - & 2 \\
\hline
\end{tabular}

